\title{
Non-rigid registration between brain CT images and MRI brain atlas by combining grayscale information, point correspondence on the midsaggital plane and brain surface matching
}

\author{
Aili Gao \\ Shenzhen Institutes of Advanced Technology \\ Shenzhen, China \\ al.gao@siat.ac.cn
}

\author{
Mingyang Chen ${ }^{1}$, Qingmao $\mathrm{Hu}^{1,2}$ \\ ${ }^{1}$ Shenzhen Institutes of Advanced Technology \\ ${ }^{2}$ Shenzhen Key Laboratory for Neuro-Psychairtric \\ Modulation \\ Shenzhen, China \\ \{my.chen1, qm.hu\}@siat.ac.cn
}

\begin{abstract}
This paper presents an image registration method to warp brain atlas of magnetic resonance image (MRI) onto computed tomography (CT) brain images. A new deformable model is proposed to add constraints on corresponding midsagittal planes (MSP). The point correspondences in the MSPs are built up from registered MSPs of the brain CT images and the atlas. The object function is a combination of mutual information, point correspondences between MSPs, and correspondence between brain boundaries. The parameters of deformable model are optimized using an iterative strategy: maximization of mutual information (MI) to find the deformable field, assigning point correspondence of MSPs to modify the deformable field, and Gaussian smoothing to make the deformable field continuous and smooth. To speed up, we adopt a multi-resolution scheme. Experiments show that the proposed method yields good results especially at the region around MSP.
\end{abstract}

Keywords-component; Registration; Feature-constrain; Multimodal; Midsagittal plane.

\section{INTRODUCTION}

Multimodal image registration is not only a key task in medical image processing but also plays an important role in many clinical applications. Since the images from different modalities are able to generate complementary information, we often need to fuse these images to show different aspects of tissue morphology or combine the tissue metabolism with its spatial location relative to anatomical structures. In this paper, we use brain CT images and MRI brain atlases as the input images of registration. The registration can be used to detect disease, approximate functional areas, segment specific structures and integrate different information of the specific structures [1].

Multimodal image registration methods can be classified into two main categories: feature-based and intensity-based methods. Feature-based methods use anatomical information of the image. On the contrary, intensity-based registration methods rely on the images intensities and ignore the anatomical knowledge. In order to combine the advantages and overcome the problems of both methods, a hybrid method has been explored in the literatures: combining anatomical and intensity features in the registration process $[2,3]$. The proposed method is a hybrid one.
In this work we mainly focus on two aspects of research. Firstly, we extract the MSP of the brain images and build the point correspondences as the constraints of transformation model. There are publications to build feature correspondences through feature points extraction and matching using SURF or SIFT algorithms [4, 5]. But this kind of algorithms is not suitable for multimodal images. In our method, we use the registration result of MSP images to get the feature point correspondences. MSP is the plane separating both hemispheres, which is one of the most important anatomical features in the human brain. Secondly, we establish a feature-constrained MI deformable model and optimize the parameters indirectly. We also add a new brain surface constraint to refine the transformation. Because feature-constrained MI deformable registration is a kind of constrained optimization, it is difficult to solve directly. So, we divide the optimization into two steps: maximize the intensity similarity and satisfy the constraints. The two steps are implemented alternately until convergence.

\section{METHODS}

\section{A. Preprocessing}

The preprocessing includes skull-stripping and affine registration. Since the MRI atlas we used is without skull, so we strip the skull of the CT brain images before registration [6]. Then we use affine registration to correct for global differences in position, orientation and scale between the reference images (CT images) and the floating images (the T1-weighted MR images corresponding to the atlas). In the process of affine registration, we choose MI as the metric and use Gradient Descent Trust Region Optimizer to optimize the transformation parameters [7].

\section{B. Feature points matching}

In most existing method, feature correspondences are built up by feature point extraction and matching. In this paper, we construct feature point correspondences in the MSPs through registration. We choose the method proposed in [8] based on local symmetry and outlier removal to extract the MSP of the brain. After extracting the MSPs of the CT data and atlas, we register them using non-parametric MI deformable model to construct feature point correspondences. After the registration, we can gain a displacement field $V$ of 
the two MSPs, which defines a displacement vector for each voxel $x$ of the MSP of the atlas with respect to that of the CT data.

$$
T(x)=x+V(x)
$$

$T(\bullet)$ is the image transformation function. $T(x)$ can be considered as the point in the warped floating image. $V(x)$ can be considered as the correspondences between the warped floating image and the original floating image. We can also consider the $V(x)$ as the point correspondences between the reference image and the original floating image. In order to use $V(x)$ to restrict the transformation in the next step, we must map $V(x)$ into 3D space and is still denoted as $V(x)$ for simplicity using the relationship between the MSP and the corresponding volumetric image. Fig. 1 shows the result of the 2D image registration.

\section{Deformable registration constrained by MSP}

In this step, a non-parametric spatial MI deformable model is chosen for transformation. In addition to the MSP constraint we also add a new restriction to refine the registration. The new restriction is the brain surface constraint to match the surface of the two brain images. The brain surface constraint is realized by transforming the reference and floating images into binary images and adding the sum of squared differences (SSD) of the binary brain images into metric to confine the transformation. The modified cost function is:

$$
E=\underbrace{-M I(F, M \circ U)}_{\text {similarity }}+\underbrace{\left\|F_{b}-M_{b}\right\|^{2}}_{\text {surface-constrant }}+\underbrace{\mu \sum_{x \in S}\|U(x)-V(x)\|^{2}}_{\text {feature-constraint }}+\underbrace{\lambda\|\nabla U\|^{2}}_{\text {smooth-constrint }}
$$

where $F$ is the reference image , $M$ is the floating image, $F_{b}$ and $M_{b}$ are respectively the binary images of the reference and floating image, $U$ is the displacement field making the cost function stationary. $\|\nabla U\|^{2}$ is the smooth constraint, $V(x)$ represents the feature points correspondences gained from last step, $\lambda$ and $\mu$ are weighting factors, “ $\circ$ ” represents composite transformation. $M I(F, M \circ U)$ is the MI of reference image $F$ and the floating image $M$ [9] defined as follows:

$$
M I(F, M \circ U)=H(F)+H(M \circ U)-H(F, M \circ U)
$$

$H(\bullet)$ represents entropy .

In order to search the optimal value of cost function, we adopt an indirect method which alternately maximize the intensity similarity and satisfy the constraints. The displacement field is refined through iterations until convergence described as follows:

Step1. Given the displacement field $U^{n-1}$, compute a correspondence update field $u_{1}^{n}$ at the nth iteration by maximizing $M I(F, M \circ U)$ with respect to $u_{1}^{n}$
Step2. Compute a correspondence update field $u_{2}^{n}$ by minimizing $\left\|F_{b}-M_{b}\right\|^{2}$

Step3. The integrated update field $u$ is computed as: $u=a u_{1}^{n}+b u_{2}^{n} \cdot a, b$ are weighting factors

Step4. Smooth the update field $u^{n}$ using Gaussian smoothing kernel

Step5. Let $\left.U^{n}=U^{n-1} \circ \exp \left(u^{n}\right)\right)$ Here we use the diffeomorphic update scheme

Step6. In the feature point $X$, let $U^{n}(x)=V(x)$

Step7. Smooth the displacement field $U^{n}$ using Gaussian smoothing kernel

Step8. Repeat steps 1 to 7 until convergence

A multi-resolution scheme is used for higher computational speed. In our method we use 3 levels from coarse to fine to search the optimal registration.

\section{RESULT}

To evaluate the performance of the proposed method, we apply our method on 20 brain CT scans and MRI atlas. The MRI atlas is from Laboratory of Neuro Imaging, UCLA. We choose the ICBM 452 atlas, which is an average of T1weighted MRIs of normal young adult brains. The evaluation is based on volume overlap ratio, and to measure how well the MSPs are aligned after registration.

We chose Dice coefficient and Hausdorff displacement to evaluate the volume overlap ratio of two images. Tables 1 and 2 show the results of Dice coefficient and Hausdorff displacement, respectively. Table 1 and Table 2 compute the Dice coefficient and Hausdorff displacement in four conditions: registration result based on MI only, MI plus MSP constraint, MI plus surface constraint, and MI plus both the brain surface and MSP constraints.

TABLE I.

\begin{tabular}{|c|c|c|c|c|}
\hline & MI only & $\begin{array}{c}\text { MI Plus } \\
\text { MSP }\end{array}$ & $\begin{array}{c}\text { MI Plus } \\
\text { surface }\end{array}$ & $\begin{array}{c}\text { MI Plus } \\
\text { both }\end{array}$ \\
\hline Mean & 0.974 & 0.976 & 0.978 & 0.979 \\
\hline $\begin{array}{c}\text { Standard } \\
\text { Deviation }\end{array}$ & 0.010 & 0.009 & 0.008 & 0.007 \\
\hline
\end{tabular}

TABLE II.

\begin{tabular}{|c|c|c|c|c|}
\hline & MI only & $\begin{array}{c}\text { MI Plus } \\
\text { MSP }\end{array}$ & $\begin{array}{c}\text { MI Plus } \\
\text { surface }\end{array}$ & $\begin{array}{c}\text { MI Plus } \\
\text { both }\end{array}$ \\
\hline Mean & 0.254 & 0.239 & 0.223 & 0.219 \\
\hline $\begin{array}{c}\text { Standard } \\
\text { Deviation }\end{array}$ & 0.259 & 0.248 & 0.248 & 0.248 \\
\hline
\end{tabular}

According to Table 1 and Table 2, we find that after the registration, the reference and floating images have been registered very well in the sense of brain volume overlap ratio. We also find that the registration results with constraints are more accurate than the results without constraints and the difference between them is significant according to the result of paired T test. Fig. 2 shows the result more intuitively. 
In this paper, we use the MSP of the brain as the constraint with the assumption that the MSP is a plane. Therefore the registration result must conform to the assumption too. Now we identify MSP correspondences to measure how well they are aligned after the registration using the method proposed in this paper compared with the registration result without the MSP constraint. The result is shown in Figure 3.

From the demarcated region in Fig. 3, we can find that the MSP of warped floating image in (c) is well aligned with the fixed image. But in (d), the MSP of the atlas brain is no more a straight line. It has been distorted to a curve.

\section{CONCLUSION}

We have described a new hybrid registration method for multimodal registration of CT brain images and MRI atlas. The method is proved to be accurate and robust. The future work will focus on applying other constraints into the transformation model and establishing more accurate transformation model.

\section{ACKNOWLEDGMENT}

This study has been supported by the Introduced Innovative R\&D Team of Guangdong Province "Robot and Intelligent Information Technology", Shenzhen Key Basic Research Project JC20100527030A, and the Joint Key Grant of National Science Foundation of China with Guangdong ProvinceU1201257.

\section{REFERENCES}

[1] Antoine MJB, Viergever MA, A survey of medical image registration, Medical Image Analysis 1998;2: 1-36.

[2] Hellier P, Barillot C, Coupling dense and landmark-based approaches for nonrigid registration, IEEE Transactions on medical imaging 2003; 22(2):217-227.

[3] Johnson HJ, Christensen GE, Consistent Landmark and IntensityBased Image Registration, IEEE Transactions on medical imaging 2002; 21(5):450-461.

[4] Chen J, Tian J, Rapid multi-modality preregistration based on SIFT descriptor, In Proceedings of the 28th Annual International Conference of the IEEE Engineering in Medicine and Biology Society 2006:1437-1440 .

[5] Han X, Feature-constrained nonlinear registration of lung CT images, Fourth Edition of the Grand Challenges in Medical Image Analysis 2010: 63-72.

[6] Hu QM, Qian GY, Aziz A, Nowinski WL,Segmentation of brain from computed tomography head images, Proceedings of the 2005 IEEE Engineering in Medicine and Biology 27th Annual Conference: 1551 155-4.

[7] Brooks R, Efficient and Reliable Methods for Direct Parameterized Image Registration,Ph.D thesis, McGill University, 2008. 1.

[8] Hu QM, Nowinski WL, A rapid algorithm for robust and automatic extraction of the midsagittal plane of the human cerebrum from neuroimages based on local symmetry and outlier removal, NeuroImage 2003; 20: 2153-2165.

[9] Wells III WM,Viola P, Atsumi H, Nakajima S, Kikinis R, Multimodal volume registration by maximization of mutual information, Medical Image Analysis 1996; 1(1):35-51.

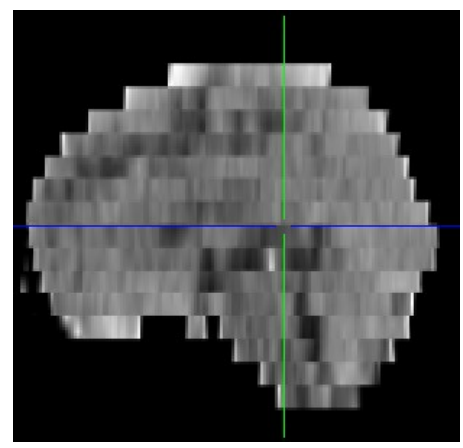

(a)

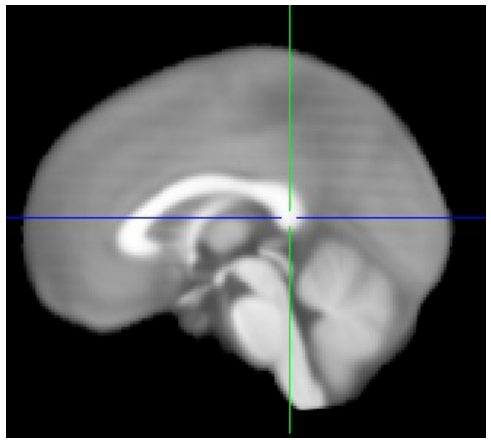

(b)

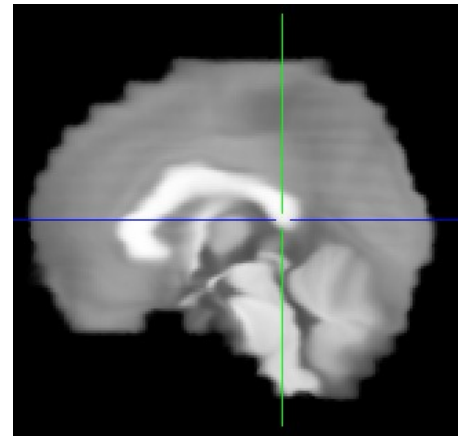

(c)

Figure 1. (a) The MSP extracted from the CT brain image (b) The MSP extracted from the MRI atlas (c)The registered MSP of the atlas (b).

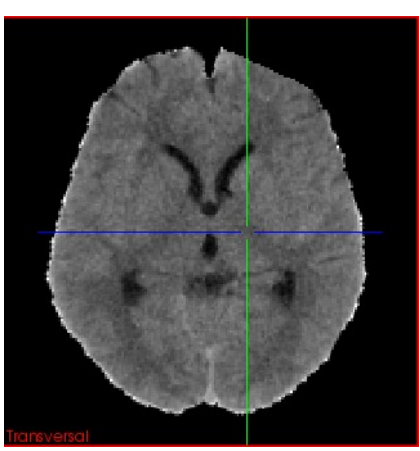

(a)

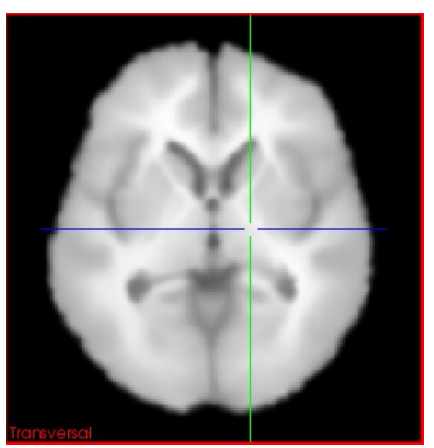

(b)

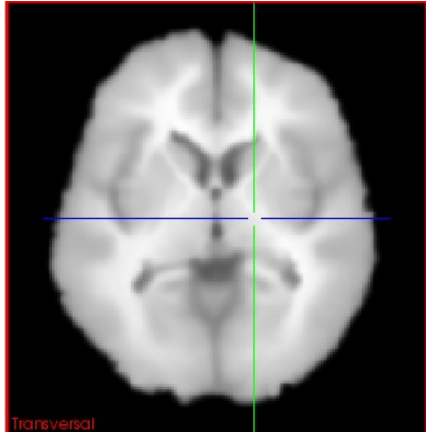

(c)

Figure 2. (a)The CT image (b)The final result with surface constraint (c)The final result without surface constraint 


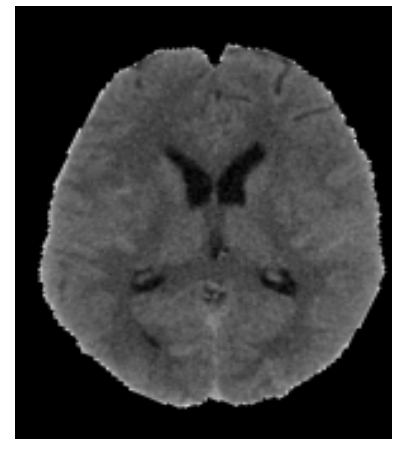

(a)

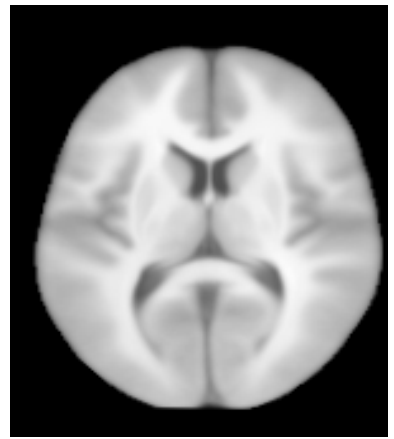

(b)

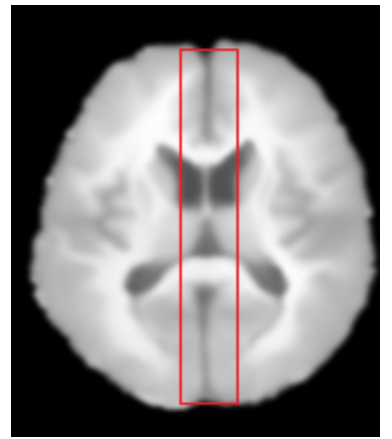

(c)

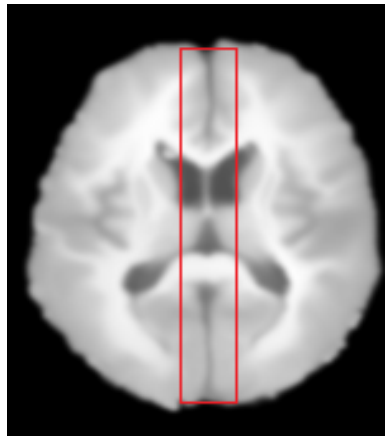

(d)

Figure 3. (a)The CT image (b)The result after the affine registration (c)The final result with MSP constraint (d)The final result without constraint 